\title{
OPEN Evidence of an epidemic spread of KPC-producing Enterobacterales in Czech hospitals
}

\author{
Lucie Kraftova ${ }^{1,2}$, Marc Finianos ${ }^{1,2}$, Vendula Studentova ${ }^{1,2}$, Katerina Chudejova $a^{1,2}$, \\ Vladislav Jakubu ${ }^{3,4}$, Helena Zemlickova ${ }^{3,4}$, Costas C. Papagiannitsis ${ }^{5,6 \square}$, Ibrahim Bitar ${ }^{1,2,6 \square}$ \&
} Jaroslav Hrabak ${ }^{1,2}$

The aim of the present study is to describe the ongoing spread of the KPC-producing strains, which is evolving to an epidemic in Czech hospitals. During the period of 2018-2019, a total of 108 KPCproducing Enterobacterales were recovered from 20 hospitals. Analysis of long-read sequencing data revealed the presence of several types of $b l a_{\mathrm{KPC}}{ }^{-c a r r y i n g}$ plasmids; 19 out of $25 b^{\prime} a_{\mathrm{KPC}}$-carrying $^{-}$ plasmids could be assigned to $R(n=12), N(n=5), C(n=1)$ and P6 $(n=1)$ incompatibility (Inc) groups. Five of the remaining bla $_{\mathrm{KPC}}{ }^{-c a r r y i n g}$ plasmids were multireplicon, while one plasmid couldn't be typed. Additionally, phylogenetic analysis confirmed the spread of $b a_{\mathrm{KPC}}$-carrying plasmids among different clones of diverse Enterobacterales species. Our findings demonstrated that the increased prevalence of KPC-producing isolates was due to plasmids spreading among different species. In some districts, the local dissemination of IncR and IncN plasmids was observed. Additionally, the ongoing evolution of $b l a_{\mathrm{KPC}}-\mathrm{carrying}$ plasmids, through genetic rearrangements, favours the preservation and further dissemination of these mobile genetic elements. Therefore, the situation should be monitored, and immediate infection control should be implemented in hospitals reporting KPC-producing strains.

Carbapenem-resistant Enterobacterales (CRE) incidence have increased, causing worldwide public-health concern due to their rapid global dissemination and limited treatment options. Carbapenemases are enzymes able to hydrolyse almost all $\beta$-lactam antibiotics including carbapenems, one of the last drugs of choice. Carbapenemases are divided into different groups depending on their structure and hydrolytic activity ${ }^{1}$. Klebsiella pneumoniae carbapenemase (KPC) is the most predominant $\beta$-lactamase of class A carbapenemases. The KPC-type carbapenemases hydrolyse a wide variety of $\beta$-lactam antibiotics such as cephalosporins, penicillins and carbapenems ${ }^{2}$.

The $b l a_{\mathrm{KPC}}$ gene was first identified in 1996 in North Carolina, USA, harboured by a K. pneumoniae isolate ${ }^{3}$. Later reports presented the monoclonal dissemination of KPC-producing isolates across America that was attributed to sequence type 258 (ST258) K. pneumoniae, as the predominant lineage ${ }^{4}$. Subsequently, KPC producers emerged in European countries, becoming highly endemic in some countries, especially in Greece and Italy ${ }^{5,6}$. Other European countries had confirmed very few cases of $b l a_{\mathrm{KPC}}$ up to 2013 according to the EuSCAPE project ${ }^{7}$. Even though KPC-type carbapenemases have been mostly associated with K. pneumoniae isolates, there are also reports of other bacterial species harbouring $b a_{\mathrm{KPC}}$-like genes, including Escherichia coli, Citrobacter freundii, Klebsiella oxytoca and other Enterobacterales, and Pseudomonas aeruginosa $a^{8,9}$.

The $b l a_{\mathrm{KPC}}$-like genes are most commonly found on the $10 \mathrm{~kb}$ transposon $\mathrm{Tn} 4401$ and its isoforms ${ }^{10,11}$. Until now, there are 9 isoforms of $\operatorname{Tn} 4401$ (a to i) ${ }^{12}$. Due to the high mobilization efficiency of $\operatorname{Tn} 4401, b l a_{\mathrm{KPC}}$-like genes have been identified on several plasmids belonging to different incompatibility (Inc) groups ${ }^{13}$.

In the Czech Republic, the first case of KPC-producing K. pneumoniae was identified in $2009^{14}$. This strain producing KPC-2, was collected from a patient repatriated from a hospital in Greece. Shortly after the first report, an outbreak of KPC-3-producing K. pneumoniae, belonging to ST512, was observed in another Czech hospital, with the index case being a patient repatriated from Italy. All those isolates harboured transposon isoform Tn4401a, carried on IncFII $\mathrm{K}_{2} \mathrm{pKpQIL-like} \mathrm{plasmids}{ }^{15}$. Another ten KPC-2-producing Enterobacterales,

\footnotetext{
${ }^{1}$ Department of Microbiology, Faculty of Medicine, and University Hospital in Pilsen, Charles University, Pilsen, Czech Republic. ${ }^{2}$ Biomedical Center, Faculty of Medicine, Charles University, Pilsen, Czech Republic. ${ }^{3}$ National Reference Laboratory for Antibiotics, National Institute of Public Health, Pilsen, Czech Republic. ${ }^{4}$ Department of Microbiology, 3rd Faculty of Medicine, Charles University, University Hospital Kralovske Vinohrady and National Institute of Public Health, Prague, Czech Republic. ${ }^{5}$ Department of Microbiology, University Hospital of Larissa, Larissa, Greece. ${ }^{6}$ These authors contributed equally: Costas C. Papagiannitsis and Ibrahim Bitar. ${ }^{\boxplus}$ email: c.papagiannitsis@gmail.com; ibrahimbitar5@gmail.com
} 
mainly of the species C. freundii, were recovered in the University Hospital of Hradec Kralove (Czech Republic) ${ }^{16}$, during the period 2014-2016. Interestingly, sequencing revealed the presence of three plasmid types with the Tn4401a transposon. The first type comprised an IncR backbone and a KPC-2-encoding multidrug resistance (MDR) region, while the second type were derivatives of the first type carrying an IncN3-like segment. Finally, the third type was IncP6 plasmids sharing the same KPC-2-encoding MDR region with the two other types.

However, a significant increase in the number of KPC-producing isolates, referred to our laboratory from Czech hospitals, was observed since 2018. The aim of the present study is to describe the ongoing spread of the KPC-type producers, which is evolving to an epidemic in Czech hospitals, during the period of 2018-2019.

\section{Results}

KPC-producing Enterobacterales. During 2018-2019, a total of 490 Enterobacterales isolates with a meropenem MIC of $>0.125 \mu \mathrm{g} / \mathrm{ml}$ were referred to the National reference laboratory for antibiotics (Prague) or to the Biomedical Center (Pilsen) from 55 laboratories. All $b l a_{\mathrm{KPC}}$-positive isolates (108) were subjected to further analysis described below. Distribution of laboratories is shown in Figure S1. Among them, 26 of the isolates were identified to be K. pneumoniae, 24 were identified to be $C$. freundii, 18 were identified to be Enterobacter cloacae complex, 14 were identified to be Proteus mirabilis, 11 were identified to be Morganella morganii and 10 were identified to be $E$. coli. The five remaining KPC-producing isolates belonged to the bacterial species, Citrobacter farmeri $(\mathrm{n}=1)$, Enterobacter aerogenes $(\mathrm{n}=1)$, K. michiganensis $(\mathrm{n}=2)$ and Klebsiella variicola $(\mathrm{n}=1)$ (Figure S2).

Analysis of short-read sequencing results. Forty-nine out of $108 \mathrm{KPC}$ producers, selected as representatives of all different hospitals, bacterial species and susceptibility profiles, were characterized by short-read sequencing using MiSeq (Illumina) platform. Based on short-read data, 44 of the 49 sequenced isolated harboured the $b l a_{\mathrm{KPC}-2}$ allele (Table S1), while the five remaining isolates carried the $b l a_{\mathrm{KPC}-3}$ gene. The $b l a_{\mathrm{KPC}-3}$ allele was identified among $3 \mathrm{~K}$. pneumoniae, $1 \mathrm{~K}$. michiganensis and 1 E. coli isolates. Beside species-specific chromosomal $\beta$-lactamases, most of the clinical isolates also carried genes encoding OXA-1/9 oxacillinases $(\mathrm{n}=37)$ and/ or TEM-1 penicillinases $(\mathrm{n}=34)$. The bla $_{\mathrm{CTX}-\mathrm{M}-15}$ gene was found among 2 Enterobacter and $5 \mathrm{~K}$. pneumoniae isolates, while 4 out of 5 P. mirabilis harboured the bla $a_{\mathrm{CTX}-\mathrm{M}-14}$ gene. Additionally, 4 out of 7 Enterobacter isolates cocarried the carbapenemase-encoding gene $b{ } a_{\mathrm{VIM}-4}$. All sequenced isolates exhibited a wide variety of resistance genes conferring resistance to aminoglycosides, sulfonamides, trimethoprim, macrolides, streptogramin B, fosfomycin (low-level resistance), fluoroquinolones, chloramphenicol, tetracyclines, and/or rifampicin (Table S1).

WGS data revealed that C. freundii isolates belonged to sequence types ST65 $(n=6)$, ST580 $(n=3)$, ST98 $(\mathrm{n}=2)$ and ST8 $(\mathrm{n}=1)$ (Table S1). ST98 C. freundii isolates producing KPC-2 carbapenemase were previously recovered from critically ill patients hospitalized in Germany ${ }^{17}$, while ST8 C. freundii expressing a VIM-4 isoenzyme were identified in Poland ${ }^{18}$, in 2013. On the other hand, the novel ST580 was a single allele variant of ST142, which was previously associated with KPC-2 production in isolates from the University Hospital of Hradec Kralove (Czech Republic) ${ }^{16}$. The isolates belonging to E. cloacae complex were assigned to ST133 $(n=4)$ and ST421 $(\mathrm{n}=3)$, which haven't been previously associated with the production of KPC-2 carbapenemase. Additionally, in silico hsp60 typing of the genome sequences showed that four Enterobacter isolates belonged to the species Enterobacter hormaechei ${ }^{19}$. The K. pneumoniae isolates included eight STs. Seven KPC-2-producers were distributed in ST101 $(\mathrm{n}=4)$ and ST11 $(\mathrm{n}=3)$. The remaining KPC-2-producing K. pneumoniae isolates belonged to unique STs (ST13, ST17 and ST147), while the K. pneumoniae isolates, which produced the KPC-3 enzyme, were ST307, ST512 and ST846. ST11, ST101, ST147, ST307 and ST512 have been previously associated with the spread of KPC resistance mechanism and have been considered as 'high risk clones ${ }^{20,21}$. Finally, the E. coli and K. michiganensis (closely related to K. oxytoca) isolates were assigned to diverse STs, as shown in Table S1. Since MLST schemes do not exist for M. morganii and P. mirabilis isolates, phylogenetic clusters for the respective isolates were determined based on core-genome alignment (see below), using the Harvest suite ${ }^{22}$.

Characterization of bla $\boldsymbol{K}_{\mathrm{KPC}}$-carrying genetic units. Based on short-read data, $25 \mathrm{KPC}$-producing isolates were selected to be sequenced by Sequel I platform, in an attempt to close plasmid sequences. All the 25 isolates showed resistance to cephalosporins and ertapenem while (except for $P$. mirabilis isolates) remained susceptible to colistin. Some variation in MIC values were noticed, however it is due to the different antibiotic resistance genes content found in each isolate (Table S3). Analysis of long-read sequencing data revealed the presence of several $b l a_{\mathrm{KPC}}$-carrying plasmid sequences belonging to different Inc groups and presenting diverse sizes (Table S2). Based on PlasmidFinder analysis of plasmid sequences, 19 out of 25 bla $_{\mathrm{KPC}}$-carrying plasmids could be assigned to $\mathrm{R}(\mathrm{n}=12), \mathrm{N}(\mathrm{n}=5), \mathrm{C}(\mathrm{n}=1)$ and P6 $(\mathrm{n}=1)$ incompatibility (Inc) groups (Figure S3). Five of the remaining $b l a_{\mathrm{KPC}}$-carrying plasmids were multireplicon, while one plasmid couldn't be typed by the database. All plasmids, except IncN replicons, contained the Tn4401a isoform of the Tn4401 transposon, which is similar to that described in plasmid pNYC, lacking $100 \mathrm{bp}$ upstream of $b l a_{\mathrm{KPC}}$ gene $^{11}$.

Three out of $12 b l a_{\mathrm{KPC}}$-carrying plasmids, belonging to IncR group, were $\bumpeq 54 \mathrm{~kb}$ in size, while the nine remaining IncR plasmids sized $\simeq 89 \mathrm{~kb}$. The IncR plasmids that were $\simeq 54 \mathrm{~kb}$ in size were derivatives of the IncR KPC-2-encoding plasmid pCfr-31816cz (Fig. 1a), which was characterized during an outbreak of KPC-2-producing Enterobacterales in a Czech hospital (Hradec Kralove) ${ }^{16}$. However, they differed from pCfr-31816cz by the presence of an additional 9232-bp sequence (nt 7286 to 16,517; GenBank accession no. CP070521) encoding CcdAB toxin-antitoxin system, and IncFIIA RepA and Ssb proteins. On the other hand, the IncR plasmids that were $\backsim 89 \mathrm{~kb}$ in size showed high degrees of similarity to each other and to the previously described plasmid pCfr-36049cz (Fig. 1b). Plasmid pCfr36049cz was characterized during the KPC-2 outbreak that took place in Hradec Kralove ${ }^{16}$, during 2014-2016. Similar to pCfr-36049cz, the latter plasmids were fusion derivatives of IncR 
(a)

(b)
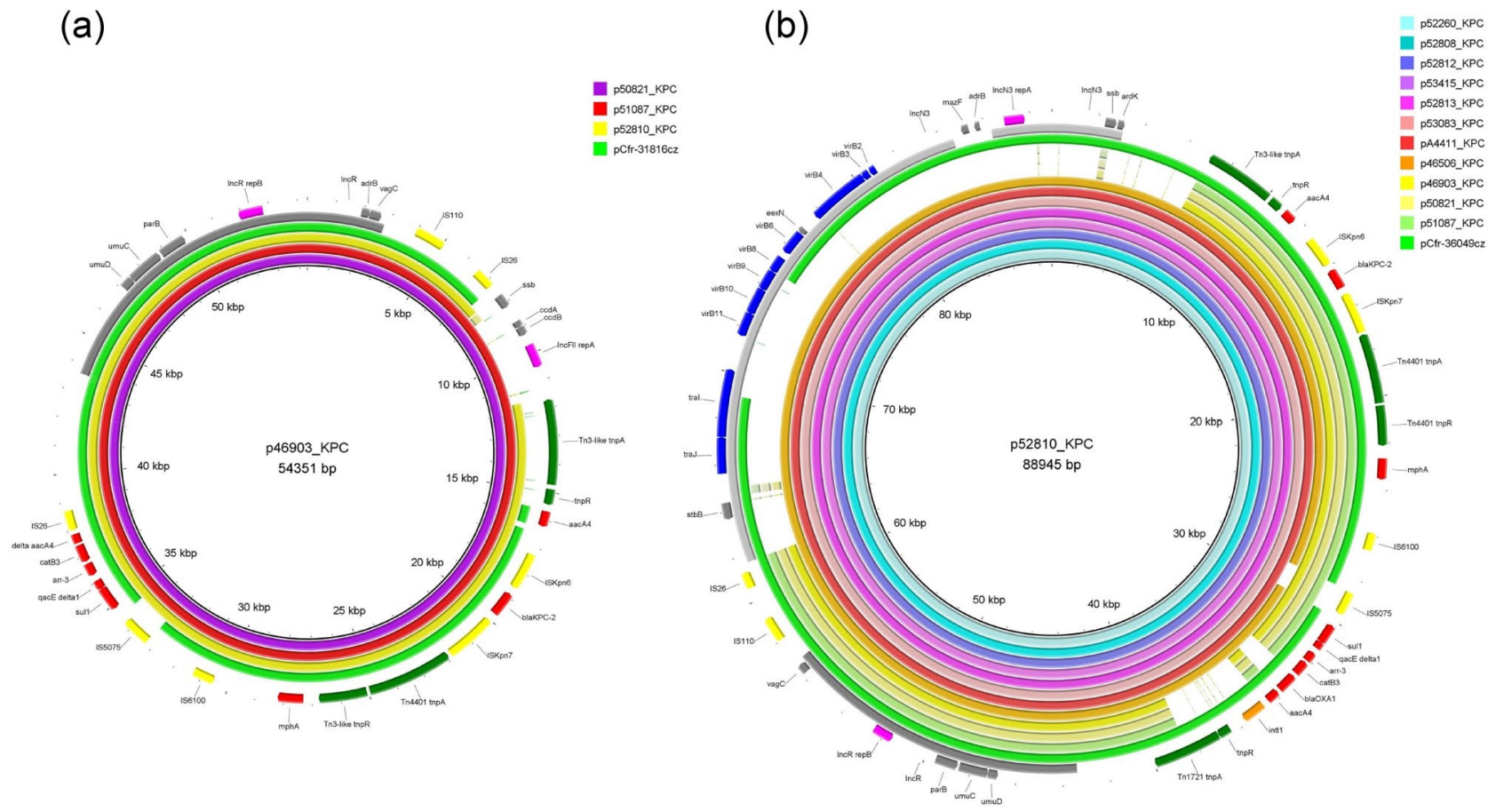

Figure 1. BRIG comparison of IncR KPC-encoding plasmids characterized from Enterobacterales isolates recovered from Czech hospitals.

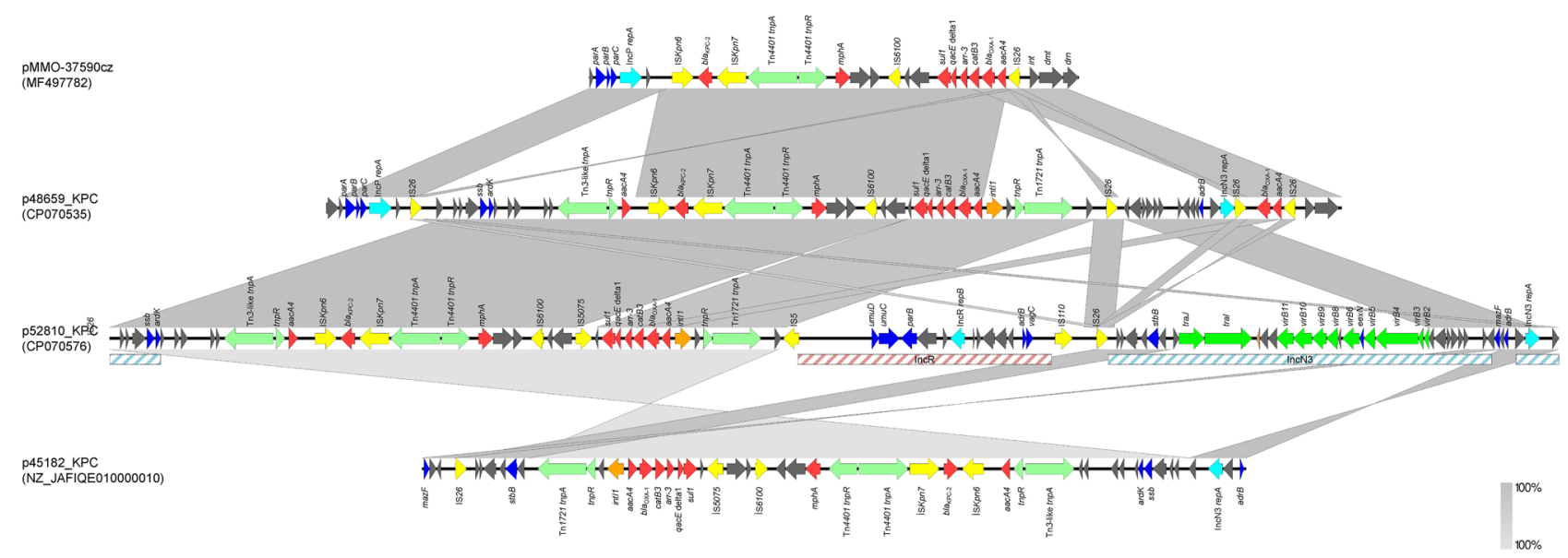

Figure 2. Linear comparisons of the KPC-encoding plasmids p48659_KPC and p45182_KPC. Arrows show the direction of transcription of open reading frames (ORFs). Resistance genes are shown in red. IS elements and transposases are shown in yellow and light green, respectively. intI1 genes are shaded purple. Genes encoding replication, stability and transfer systems are shown in aqua, blue and green colors, respectively. The remaining genes are shown in gray. Homologous segments (representing $\geq 85 \%$ sequence identity) are indicated by gray shading.

$b l a_{\mathrm{KPC}-2}$-positive plasmids and an IncN3-type-derived segment. However, unlike in pCfr-36049, a complete IncN3 transfer system was found, explaining the ability of pA4411_KPC, p46506_KPC, p52260_KPC, p52808_KPC, p52810_KPC, p52812_KPC, p52813_KPC, p53083_KPC and p53415_KPC to transfer via conjugation. Of note, the IncR plasmid p52813_KPC carried the $b l a_{\mathrm{KPC}-3}$ allele, indicating the ongoing evolution of the determinants encoding KPC carbapenemases.

Plasmid p48659_KPC is a fusion derivative of the p52810_KPC and pMmo-37590cz (Fig. 2). pMmo-37590cz is an IncP6 KPC-2-encoding plasmid that was also characterized during the KPC-2 outbreak in Hradec Kralove hospital ${ }^{16}$. Plasmid p48659_KPC contains a 50,603-bp sequence (nt 5917 to 56,519) encoding KPC-2, which is identical to a partial sequence of p52810_KPC. The remaining 11,723-bp sequence of p48659_KPC consists of one segment sharing extensive similarity with sequences carried by $\mathrm{pMmo}-37590 \mathrm{cz}$. This segment included the IncP6 replication gene repA, the partitioning genes, $\operatorname{par} A$, par $B$, and par $C$, and genes encoding a DNA invertase/ 


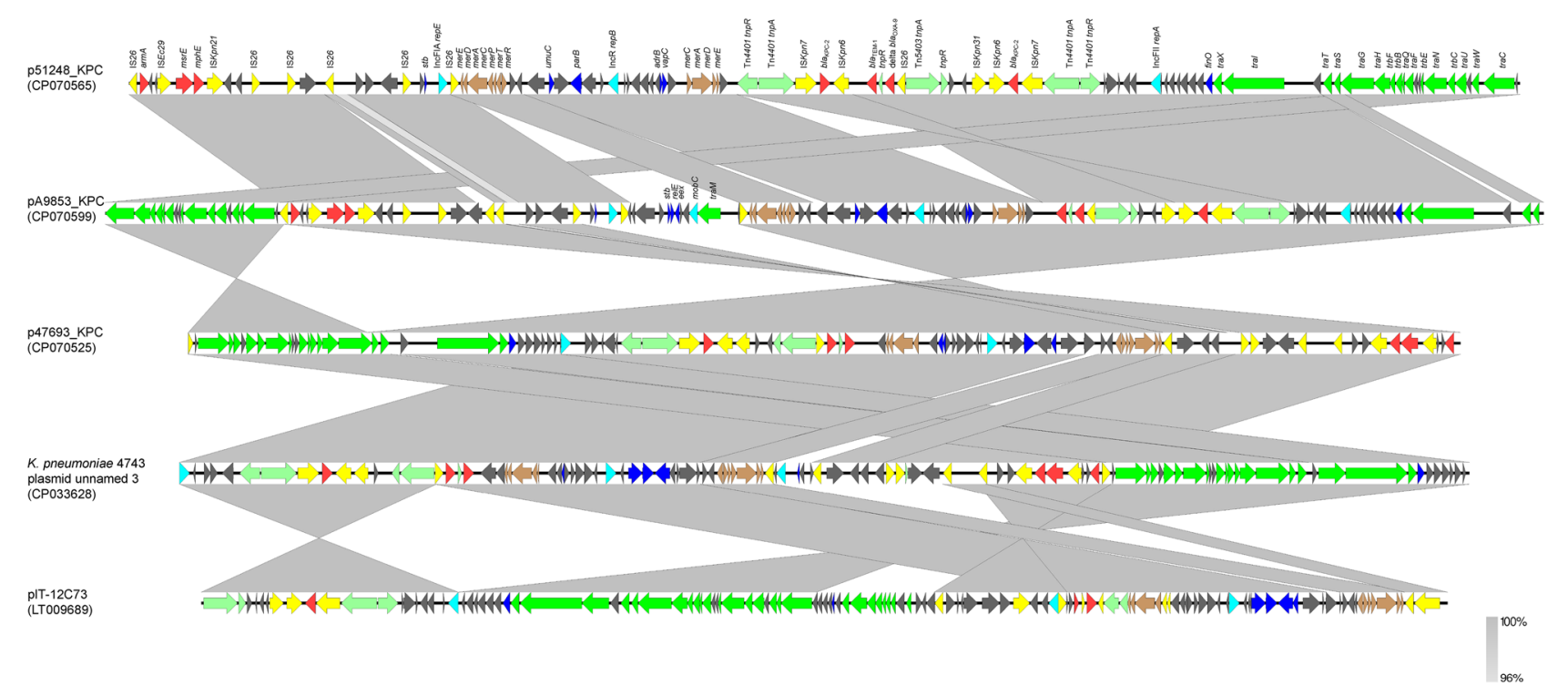

Figure 3. Linear comparisons of the multireplicon KPC-encoding plasmids p51248_KPC,pA9853_KPC and p47693_KPC. Arrows show the direction of transcription of open reading frames (ORFs). Resistance genes are shown in red, while genes involved in mercury resistance are shaded brown. IS elements and transposases are shown in yellow and green, respectively. intI1 genes are shaded purple. Genes encoding replication, stability and transfer systems are shown in aqua, blue and green colors, respectively. The remaining genes are shown in gray. Homologous segments (representing $\geq 85 \%$ sequence identity) are indicated by gray shading.

recombinase (int), a deoxymethyltransferase $(d m t)$, and a DNase $(d r n)$ of type II restriction module. Sequence analysis demonstrated that the plasmid p45182_KPC, which was not typed by PlasmidFinder, is 50,582 bp in size and is a derivative of p52810_KPC (Fig. 2). Only two differences between the two plasmids were observed. A 26,069-bp segment (nts 34,454 to 60,522 in p52810_KPC) including IncR plasmidic backbone, a Tn1721-like fragment (consisting of the 38-bp inverted repeat of the transposon, $\operatorname{tn} p A, \operatorname{tn} p R$, and $\operatorname{tn} p M$ ), and intI1 gene of the integron In37, was not present in p45182_KPC. In addition, a second fragment (nts 65,142 to 83,473 in p52810_KPC), being 18,332 bp in size, that contained vir2/3/4/9/10/11 region of IncN3-like plasmids was also absent from p45182_KPC, probably explaining the inability of the plasmid to conjugate.

The IncN $b l a_{\mathrm{KPC}-2}$-positive plasmids, which were assigned to ST15 based on the pMLST (https://cge.cbs.dtu. $\mathrm{dk} /$ services/pMLST/), comprised the plasmidic backbone and a multidrug resistance (MDR) region inserted downstream the fipA. The IncN plasmidic backbone contained a replication region (repA), a transfer system $(\operatorname{tra} A / B / C / D / N / E / O / F / G)$, a stability operon $(s t b A / B / C)$ and an antirestriction system $(\operatorname{ard} A / B)$. The MDR region of IncN plasmids, which ranged from 21,011 to $31,420 \mathrm{bp}$ in size, harboured a Tn4401-derived fragment of 2833-bp, encoding KPC-2 carbapenemase. In comparison to Tn4401b, the Tn4401-derived sequence (designated $\mathrm{Tn} 4401 \mathrm{j}$ ) had a deletion of $217 \mathrm{bp}$ found upstream of the $b l a_{\mathrm{KPC}-2}$. The Tn4401-derived fragment was disrupted by a Tn3-like sequence, $111 \mathrm{bp}$ upstream of the $b l a_{\mathrm{KPC}-2}$. The Tn3-like sequence was composed of the inverted repeat (IR) of the transposon and the $b l a_{\mathrm{TEM}-1}$ resistance gene. A similar $b l a_{\mathrm{KPC}-2}$-carrying genetic environment has been previously identified in the IncN plasmid pCF8698_KPC2, characterized from a C. freundii strain isolated in Germany (GenBank accession no. LN610760) (Figure S4). The MDR region of the IncN plasmids exhibited additional genes conferring resistance to aminoglycosides, sulfonamides, trimethoprim, macrolides, and/or fluoroquinolones (Table S2, Figure S4).

Plasmid p49969_KPC, typed as IncC based on PlasmidFinder, exhibited highest similarity with the OXA204-encoding plasmid pCf3880 (74\% coverage, 99.87\% identity) (Figure S5). The pCf3880, which was an IncFII/ $\mathrm{FIB} / \mathrm{C}_{2}$ hybrid plasmid, was characterized from a C. freundii isolated from a hospital in Canada ${ }^{23}$. Unlike pCf3880, plasmid p49969_KPC carried the carbapenemase-encoding gene, $b l a_{\mathrm{KPC}-2}$, which resulted from the acquisition of a 29,121-bp fragment (nt 111,204 to 140,234 in GenBank accession no. CP070549) showing extensive similarity to IncN plasmid p48846_KPC. The IncN-derived fragment was bound by two copies of the insertion sequence IS26, in parallel orientation, forming a composite transposon. Plasmid p49969_KPC carried, also, the resistance genes $b l a_{\text {TEM-1B }}$, aac (6')-Im, aac(3)-IId and $a p h\left(2^{\prime \prime)}-I b\right.$.

Plasmids pA9853_KPC, p47693_KPC and p51248_KPC, which were characterized from ST101 K. pneumoniae isolates (Table S2), exhibited extensive similarity to pIT- $12 \mathrm{C} 73^{20}$ and plasmid unnamed 3 (GenBank accession no. CP033628) (Fig. 3). Plasmid pIT-12C73 which was also characterized from a ST101 K. pneumoniae, isolated in Italy in 2011, was a multireplicon IncFII ${ }_{\mathrm{K} 2}$-IncR KPC-encoding plasmid. Plasmids pA9853_KPC and p51248_KPC differed from pIT-12C73 by acquisition of a 3254-bp fragment containing the IncFIA repE gene. Plasmid pA9853_KPC harboured an additional 9295-bp sequence, being identical to ColE1-like plasmid pColRNAI-Kp1-1 (GenBank accession no. LC505458). Comparative analysis suggested that IS26-mediated recombination events likely played a major role in acquisition of fragments of diverse origin. Similarly, to pIT12C73, apart from $b l a_{\mathrm{KPC}-2}$, plasmids pA9853_KPC, p47693_KPC and p51248_KPC carried also bla $a_{\mathrm{TEM}-1}$, armA, 


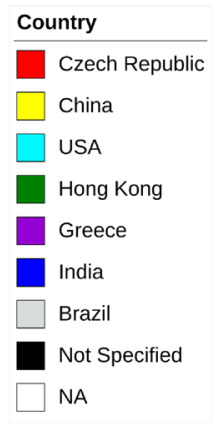

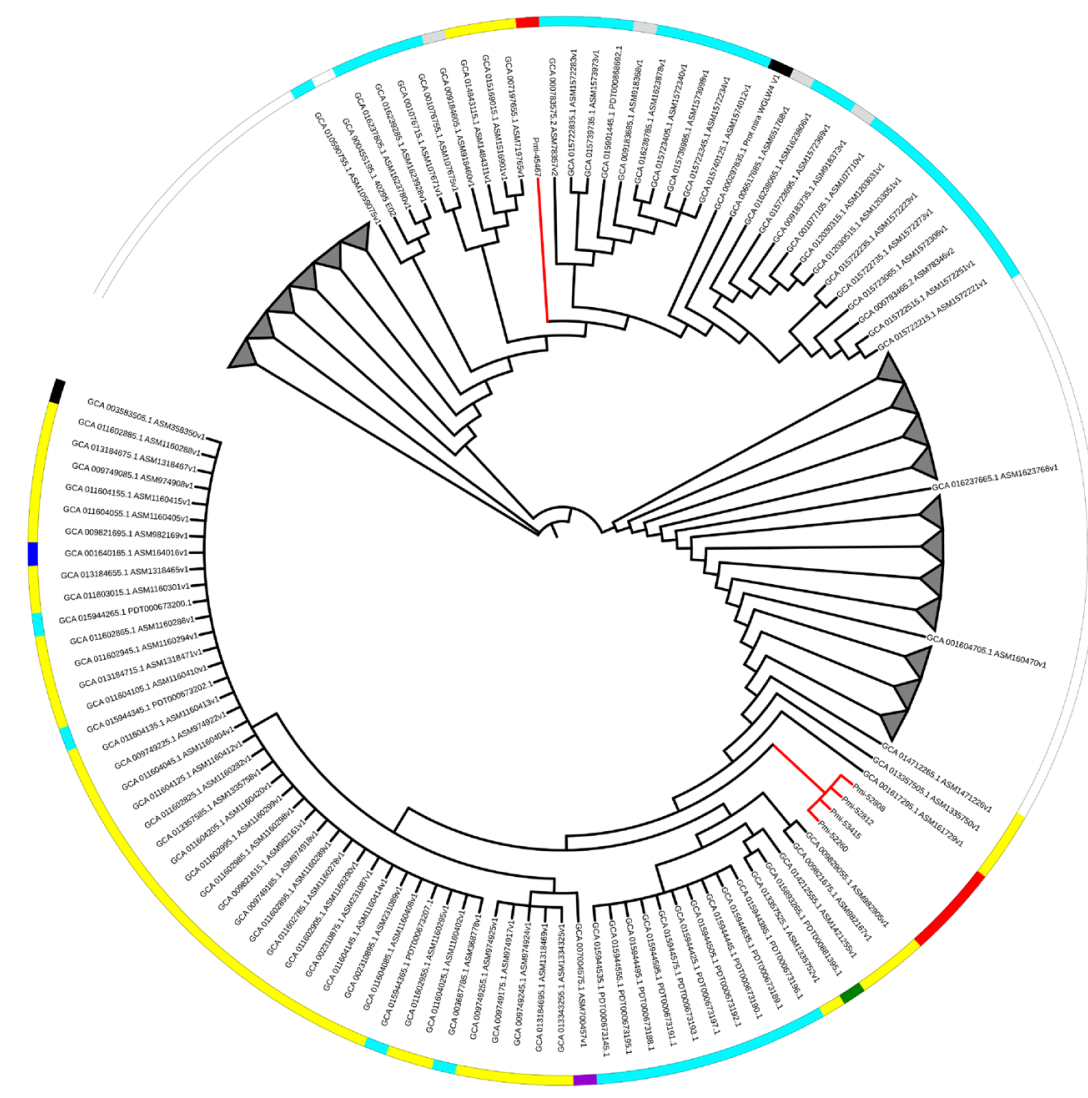

Figure 4. SNPs-based phylogeny of the five P. mirabilis isolates with 582 genomes downloaded from NCBI database. Red nodes indicate the Isolates from the study. Grey triangles indicate collapsed nodes.

$m p h E, m s r E$ resistance genes (Fig. 3). Interestingly, a duplication of the KPC-2-encoding transposon, Tn4401a, was found in plasmid p51428_KPC.

Plasmid p51483_KPC, which contained replicons FIB $_{\mathrm{K}}$ and $\mathrm{FII}_{\mathrm{K}}$, showed highest similarity to KPC-2-encoding plasmid pRIVM_C008981_1 (96\% coverage; 99.93\% identity) (Figure S6a), characterized from a K. pneumoniae isolate collected in the Dutch national surveillance ${ }^{24}$. Similarly, to pRIVM_C008981_1, apart from regions responsible for conjugative transfer $(\operatorname{traX} / I / D / T / S / G / H / F / B / Q / C / U / W / V / P / K / Y / M)$ and plasmid maintenance ( $p$ si $A / B$, par $A / B$ and $u m u D / C$ operons), p51483_KPC carried genes $s i l E / S / R / C / B / A / P$ encoding a silver exporting ATPase, $p c o A / B / C / D / R / E$ involved in copper resistance, ars $H / D / A / C / B / A / D / R$ conferring arsenic resistance, and $f e c I / R / A / B / C / D / E$ implicated in $\mathrm{Fe}(3+)$ dicitrate transport. In addition to $b l a_{\mathrm{KPC}-2}$, plasmid p51483_KPC contained $b l a_{\mathrm{TEM}-1}$, aadA2, aph(3')-Ia, catA1, dfrA12 and $m p h A$ resistance genes. Finally, plasmid p51059_KPC, isolated from a ST512 K. pneumoniae, showed high similarity to IncFII ${ }_{\mathrm{K} 2}$ KPC-2 encoding plasmids pGR-1504 ( $99 \%$ coverage; $99.97 \%$ identity) and pIT-01C22 (coverage 99\%; identity 99.96\%) (Figure S6b) characterized from two endemic settings, Greece and Italy, during 2010-2011 ${ }^{20}$. Plasmids pGR-1504 and pIT-01C22 were derivatives of the archetypal $\mathrm{IncFII}_{\mathrm{K} 2} \mathrm{KPC}$-encoding plasmid pKpQIL, originally described in the ST258 K. pneumoniae Kpn557 isolate ${ }^{25}$.

Genomic comparison and relatedness. Sequence data from the 49 sequenced isolates, have been used to investigate their genomic relatedness with global isolates and SNPs based phylogenies have been constructed accordingly. For $P$. mirabilis, the five sequenced isolates were compared against 582 genomes found in the NCBI database (Fig. 4). Four isolates (Pmi-52808, Pmi-52812, Pmi-53415 and Pmi-52260) clustered together forming a clade. These isolates were isolated from the same hospital (NY) and using Pmi-52260 as reference for SNPs detection, Pmi-52808 and Pmi-53415 shared 14 and 9 alterations (snps, del/ins), respectively, while Pmi-52812 shared 117 alterations (Table S4). Lastly, Pmi-45467, isolated in hospital HK, was relatively distant from the rest of the isolates.

Similarly, 92 M. morganii available genomes in NCBI database were downloaded to compare them with the five isolates sequenced in this study (Fig. 5). Mmo-48659, isolated in HK hospital, clustered alone in a unique node. However, it was closely related to an isolate from South Africa (790 alteration). On the other hand, four isolates (isolated from three different hospitals; Table S1) clustered together, with Mmo-51087 and Mmo-50821 isolated from the same hospital forming a subclade. For the detection of SNPs among the four isolates of this clade, Mmo-51087 was used as a reference. Mmo-50821 and Mmo-46544 showed 22 and 62 alterations, respectively, while Mmo-46903 222 alterations (Table S4). 

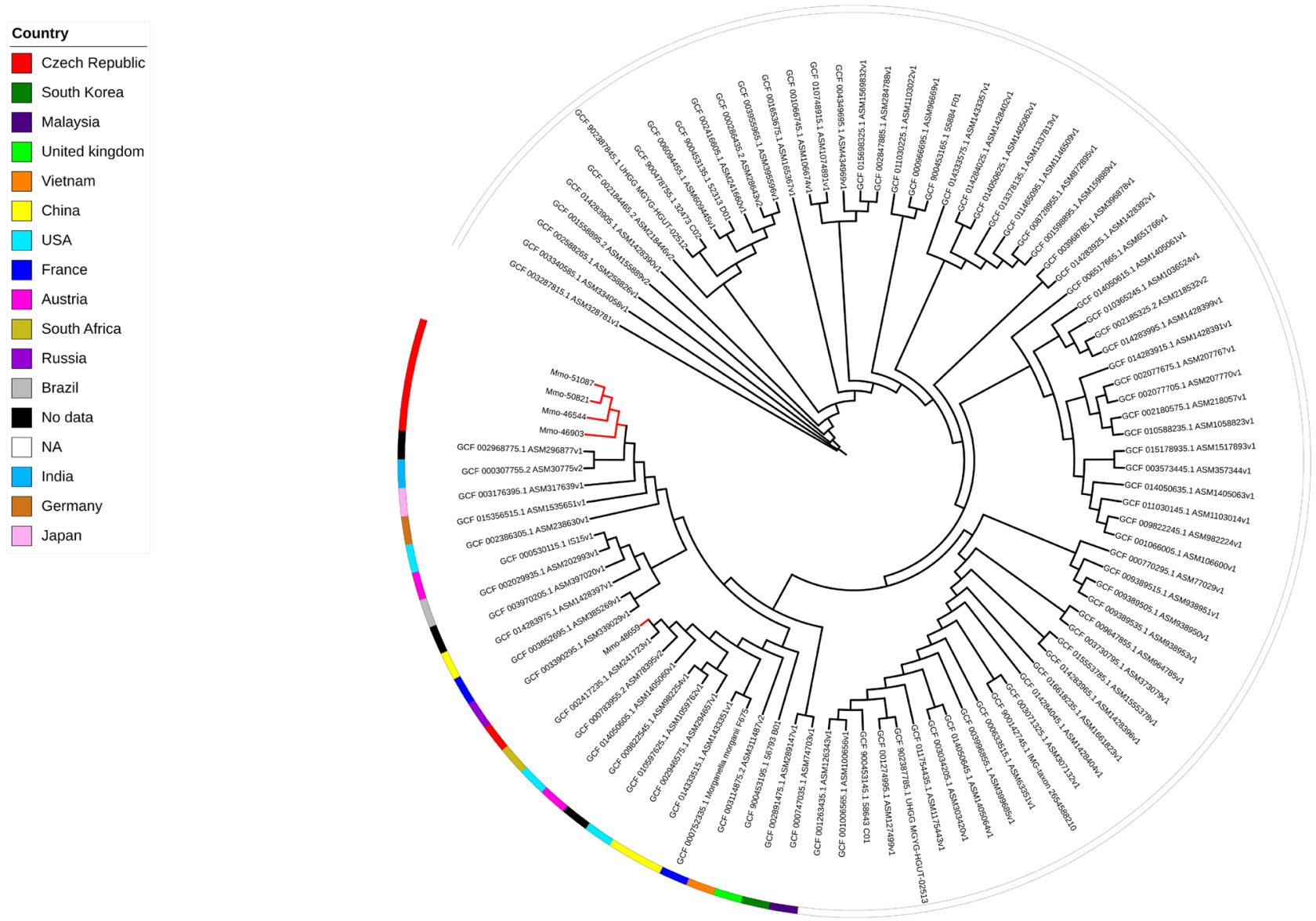

Figure 5. SNPs-based phylogeny of the five M. morganii with 92 genomes downloaded from NCBI database. Red nodes indicate the Isolates from the study.

For C. freundii, 118 genomes were downloaded from the NCBI database to compare them with the 12 sequenced isolates (Fig. 6). The genomes of three isolates (Cfr-46338, Cfr-49942 and Cfr-48658), which belonged to ST580 and were recovered from HK hospital, clustered together forming a clade. SNPs detection among these isolates showed that Cfr-49942 and Cfr- 46338 had 93 and 111 alterations, respectively, compared to Cfr- 48658 . In a closely related clade, another six genomes from ST65 isolates Cfr-50935, Cfr-48846, Cfr-51238, Cfr-47462, Cfr-48294 and Cfr-47299 clustered together. SNPs detection when compared to Cfr-50935 showed that Cfr48846 had 25 alterations while Cfr-51238, Cfr-47462, Cfr-47299 and Cfr-48294 had 47, 57, 88 and 99 respectively (Table S4). On the other hand, the genomes of the two ST98 isolates, Cfr-48736 and Cfr-49141, clustered together in a considerable distant clade. These isolates are clustered together with other ST98 C. freundii isolates from the USA and UK. SNPs detection showed that Cfr-48736 had 28 alterations when compared to Cfr-49141. Finally, the isolate Cfr-49969, which was assigned to ST8, resulted in a unique node.

For Enterobacter hormaechei, 126 genomes were downloaded from the NCBI database and were compared with the seven isolates sequenced during this study (Fig. 7). The isolates clustered in two clades. The first clade contained four isolates (Ecl-49142, Ecl-48587, Ecl-48293 and Ecl-49583). Ecl-48293 was used as a reference genome for SNPs detection among these four isolates. Ecl-49142 had 26, Ecl-48587 had 34 and Ecl-49583 had 32 alterations. All ST133 isolates recovered from Czech Republic, South Africa, Japan, Australia and Egypt were grouped in a unique cluster. Additionally, the other cluster contained the three isolates (Ecl-51693, Ecl-51846 and Ecl-52075) which were ST421. For SNPs detection, Ecl-51846 was used as a reference, showing that Ecl-52075 and Ecl-51692 had 12 and 14 alterations, respectively (Table S4).

For K. pneumoniae, 732 genomes were downloaded from the NCBI database to compare them with the 13 sequenced isolates (Fig. 8). Isolates Kpn-51835, Kpn-47158, Kpn-51483, Kpn-53027, Kpn-52813 and Kpn-51069, which were assigned to diverse STs, formed a unique distinct node each. One clade consisting of Kpn-47693, Kpn-O141 and Kpn-A9853 was in close proximity with Kpn-51248 in the neighbouring cluster. Using Kpn-47693 as a reference genome for SNPs detection, Kpn-O141, Kpn-A9853 and Kpn-51248 had 25, 22 and 100 alterations, respectively. The above isolates were grouped together with other ST101 isolates from Italy, USA, Japan, India and South Africa. The last three K. pneumoniae isolates (Kpn-52810, Kpn-A4411 and Kpn-45128) clustered together. Using Kpn-52810 as a reference genome for SNPs detection, Kpn-A4411 had 27 alterations, while Kpn-45128 exhibited 816 alterations (Table S4). Interestingly, the last isolates were clustered with isolates from China, Switzerland, India and the USA, which belonged also to ST11. 

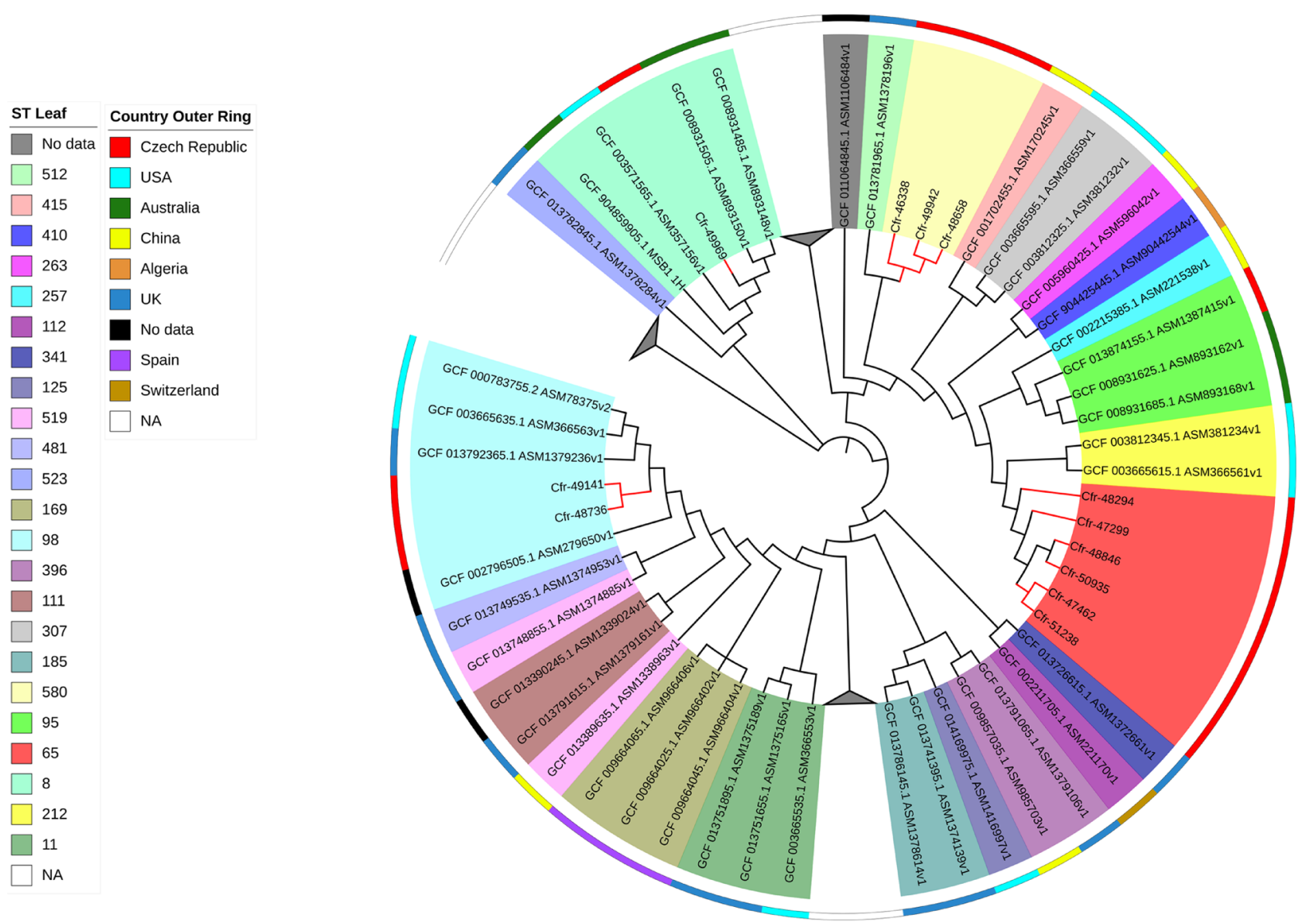

Figure 6. SNPs-based phylogeny of the 12 C. freundii with 118 genomes downloaded from NCBI database. Red nodes indicate the Isolates from the study. Grey triangles indicate collapsed nodes.

\section{Discussion}

KPC-producing Enterobacterales represent a major threat of global dimensions for public health. The current study described the change of the epidemiological situation in Czech hospitals, from the sporadic cases or outbreaks to the epidemic spread of KPC-producing isolates (Figure S1). During 2018-2019, 108 KPC producers were recovered from 22 different Czech hospitals located throughout the country. Additionally, the $b l a_{\mathrm{KPC}}$ gene was found among diverse species and clones of Enterobacterales family (Figure S2).

Phylogenetic analysis indicated that $P$. mirabilis and $M$. morganii isolates, carrying the $b l a_{\mathrm{KPC}}$-like gene, didn't exhibit close relationship with isolates characterized previously from other geographical areas. Additionally, phylogenetic analysis showed that the KPC-2-producing E. hormaechei isolates belonged to two distinct clones (Fig. 7), assigned as ST133 and ST421 based on MLST. The ST421 isolates weren't closely related with other isolates analysed, using parsnp software, while ST133 isolates clustered together with isolates from South Africa, Japan, Australia and Egypt. However, ST133 isolates, recovered from other geographical areas, weren't associated with the production of KPC-2 carbapenemase. Regarding C. freundii isolates, phylogenetic analysis revealed two main clones, which were assigned to ST65 and ST580 based on MLST. These two clones were distinct to each other and to the isolates included in the analysis. Isolates Cfr-48736 and Cfr-49141 grouped together with other ST98 isolates from the UK and USA, while the isolate Cfr-49969 was clustered with other ST8 isolates from Australia and the USA. However, the later clones have been associated with the production of KPC-2 and VIM-4 carbapenemases in Germany and Poland ${ }^{17}{ }^{18}$, respectively. On the other hand, 6 out of $13 \mathrm{~K}$. pneumoniae isolates characterized by WGS belonged to unique STs. Interestingly, parsnp phylogenetic analysis clustered these isolates with other K. pneumoniae isolates, belonging to the same STs, from worldwide. Additionally, the 7 remaining K. pneumoniae isolates belonged to two distinct clades. The later clades included ST11 and ST101 isolates from different geographical origins. Among K. pneumoniae, the 'high risk' clones, ST11, ST101, ST147 and ST512, that have been previously associated with the spread of KPC resistance mechanism were found ${ }^{20,26}$. In agreement with recent reports, those data confirm that high-risk clones, other than CC258, currently contribute to spread of KPC resistance mechanism in Europe ${ }^{24,25}$. Finally, the KPC-producing E. coli and $K$. michiganensis isolates belonged to unique STs. These findings underline the ongoing spread of the KPC resistance mechanism among different species and clones.

The analysis of the genetic units carrying the $b l a_{\mathrm{KPC}}$-like genes revealed the presence of a wide variety of plasmids involved in the spread of the KPC resistance mechanism. Some of the observed plasmid-types, like IncFII $_{\mathrm{K} 2}$ pKpQIL, IncFII $\mathrm{K}_{2}$-IncR pIT-12C73, and IncR-IncN 3 pCfr-36049cz, have been previously described to 

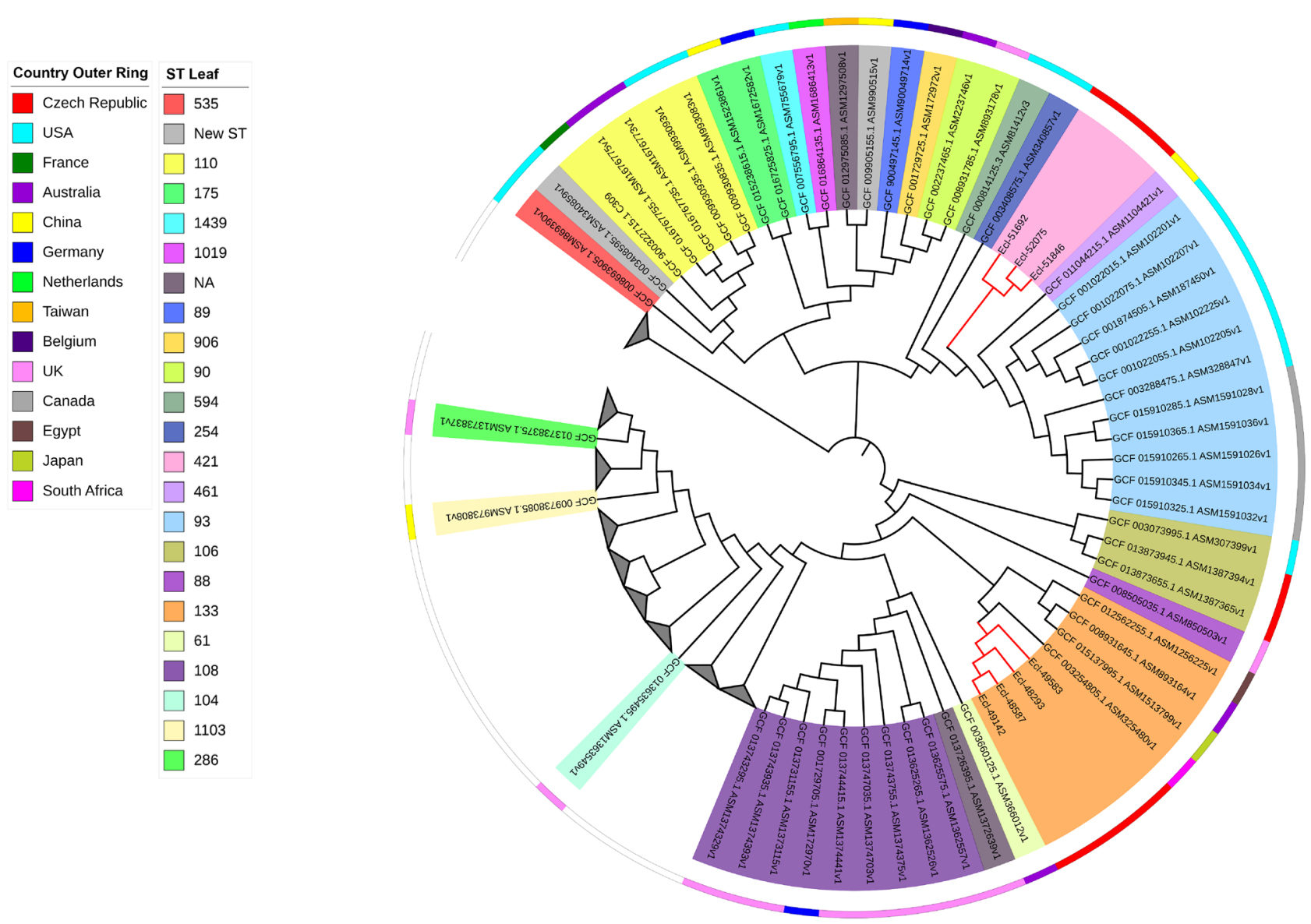

Figure 7. SNPs-based phylogeny of the seven E. homaechei with 126 genomes downloaded from NCBI database. Red nodes indicate the Isolates from the study. Grey triangles indicate collapsed nodes.

be responsible for the spread of the $b l a_{\mathrm{KPC}}$-like genes ${ }^{16,20,25}$. Additionally, some novel emerging plasmid-types, as the IncN pCF8698_KPC2 originally described from Germany (GenBank accession no. CP070521), the IncFIB ${ }_{K} /$ FII $_{K}$ PRIVM_C008981_1 firstly characterized from a Dutch collection ${ }^{24}$, and the hybrid IncFII/FIB/C $/ \mathrm{N}$ plasmid p49969_KPC characterized during this study, were identified to disseminate the $b l a_{\mathrm{KPC}}$-like genes. A few fusion derivatives of the $b l a_{\mathrm{KPC}}$-carrying plasmids described above were observed. These data verify the presence of some successful plasmid lineages spreading the KPC resistance mechanism, but also highlight the ongoing evolution of the mobile genetic elements involved in the dissemination of clinically important resistance mechanisms. For example, IncR plasmids carrying $b l a_{\mathrm{KPC}}$ genes have played a significant role in the spread of the specific resistance mechanism, in the Czech Republic. But, IncR plasmids have also been involved with the spread of other important carbapenemases, like NDM and VIM ${ }^{27,28}$. Additionally, in agreement with previous studies ${ }^{20,25,29}, \mathrm{IncF}$ plasmids are one of the major factors contributing to the worldwide spread of KPC carbapenemases. Moreover, the distribution of the different plasmid types detected suggests local dissemination with IncR plasmid spreading in middle part of the map especially in Hradec Kralove and Nymburk (Figure S3), while the IncN plasmid spreading in the North West of the Bohemian region. However, Prague seems like the melting pot, in which all plasmid families were detected, indicating the transient admission of patients from surrounding districts to Prague for specialized treatment. Moreover, this is confirmed by the fact that most of the isolated strains in Prague comes from private labs which provides services for many hospitals and long-term care facilities in and outside Prague. This route of dissemination could be explained by the spread of specific plasmid families within the same region, like IncR plasmids, or crossing the borders via travelling, like IncN plasmid from Germany.

In conclusion, our results show that the increased prevalence of KPC-producing isolates was due to plasmids being conjugative and spreading among different species and clones. Additionally, the ongoing evolution through genetic rearrangements, observed in $b l a_{\mathrm{KPC}}$-carrying plasmids, favour the preservation and further dissemination of these mobile genetic elements. Therefore, the situation should be monitored, and immediate infection control should be implemented in hospitals reported.

\section{Material and methods}

Bacterial isolates, carbapenemase production and susceptibility testing. From 2018 to 2019, National reference laboratory for antibiotics referred a total of 108 Enterobacterales isolates being PCR positive for $b l a_{\mathrm{KPC}}$. Species identification was performed by matrix-assisted laser desorption ionization-time of flight mass spectrometry (MALDI-TOF MS) using MALDI Biotyper software (Bruker Daltonics, Bremen, Germany). 


\begin{tabular}{ll} 
Country Outer Ring & ST Leaf \\
\hline$\square$ Czech Republic & $\square 11$ \\
$\square$ China & $\square 256$ \\
India & $\square 258$ \\
$\square$ USA & $\square 512$ \\
$\square$ Switzerland & $\square 1519$ \\
$\square$ NA & $\square 66$ \\
$\square$ Italy & $\square$ New ST \\
$\square$ France & $\square 2268$ \\
$\square$ Australia & $\square 491$ \\
$\square$ Iran & $\square 846$ \\
$\square$ South Korea & $\square 2998$ \\
$\square$ Iraq & $\square 2938$ \\
$\square$ Vietnam & $\square 307$ \\
$\square$ Thailand & $\square 1662$ \\
$\square$ Nepal & $\square 1731$ \\
$\square$ Sweden & $\square 273$ \\
$\square$ Canada & $\square 392$ \\
$\square$ Greece & $\square 147$ \\
$\square$ Albania & $\square$ NA \\
$\square$ Pakistan & $\square 1440$ \\
$\square$ UAE & $\square 17$ \\
$\square$ Taiwan & $\square 13$ \\
$\square$ UK & $\square 2668$ \\
$\square$ Japan & $\square 3750$ \\
$\square$ South Africa & $\square 43$ \\
$\square$ Germany & $\square 1685$
\end{tabular}

101

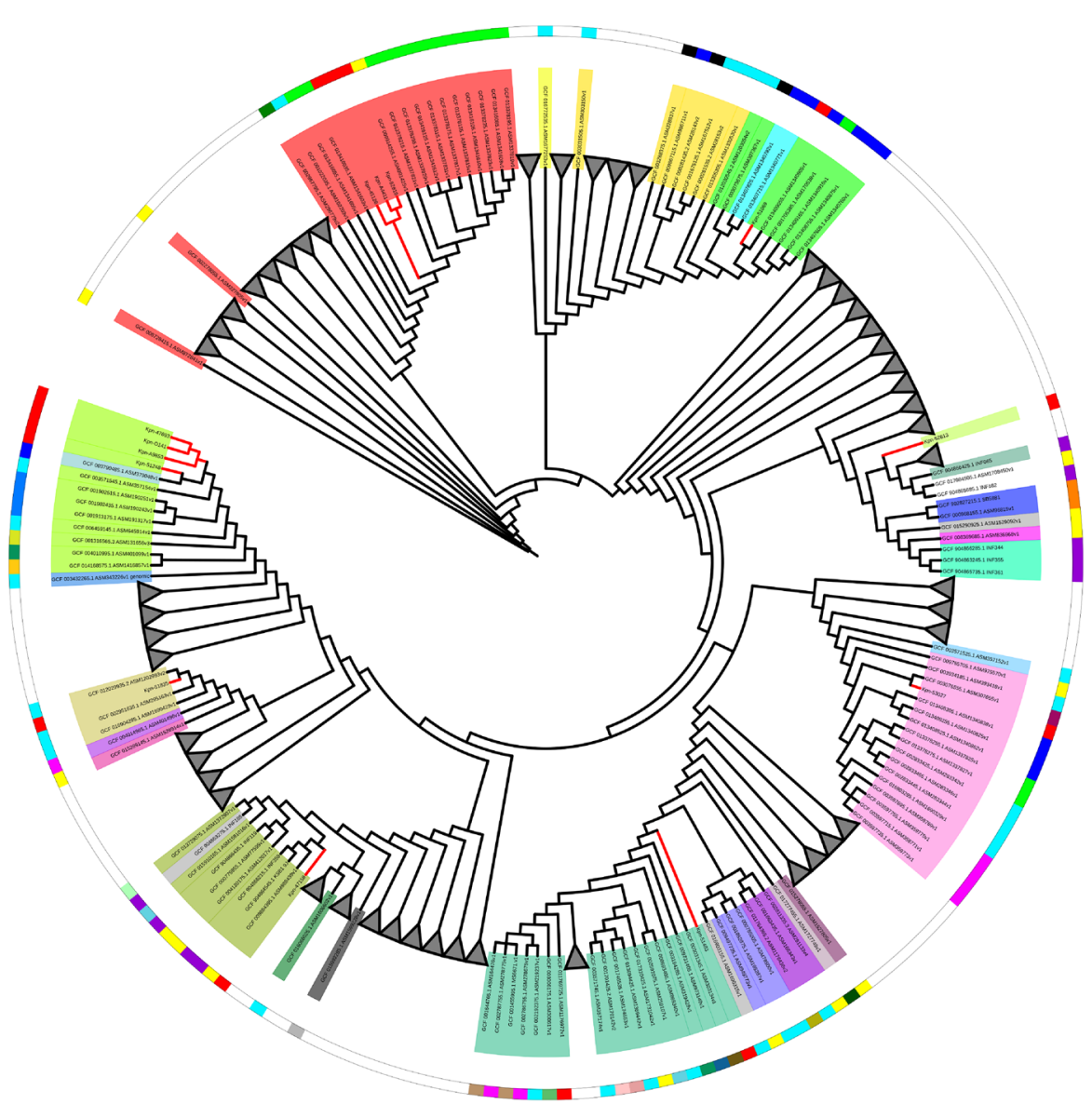

Figure 8. SNPs-based phylogeny of the $13 \mathrm{~K}$. pneumoniae with 732 genomes downloaded from NCBI database. Red nodes indicate the Isolates from the study. Grey triangles indicate collapsed nodes.

All isolates were tested for carbapenemase production by the MALDI-TOF MS meropenem hydrolysis assay ${ }^{30}$. Additionally, the presence of carbapenemase-encoding genes $\left(b l a_{\mathrm{KPC}}, b l a_{\mathrm{VIM}}, b l a_{\mathrm{IMP}} b l a_{\mathrm{NDM}}\right.$, and $b l a_{\mathrm{OXA}-48}$-like) was confirmed by PCR amplification ${ }^{11,31-33}$. Antimicrobial susceptibility was performed using broth microdilution according to European Committee on Antimicrobial Susceptibility Testing (EUCAST) guidelines. Susceptibility data were interpreted according to the criteria (version v11.0) of the EUCAST (http://www.eucast.org/).

Short-read whole genome sequencing. Forty-nine KPC-producing Enterobacterales were selected for complete sequencing, using the Illumina MiSeq platform (Illumina Inc., San Diego, CA, USA). These isolates were selected as representatives of all different hospitals, bacterial species and susceptibility profiles.

The genomic DNAs of the clinical isolates were extracted using the DNA-Sorb-B kit (Sacace Biotechnologies S.r.l., Como, Italy). Multiplexed DNA libraries were prepared using the Nextera XT library preparation kit, and 300-bp paired-end sequencing was performed on the Illumina MiSeq platform (Illumina Inc., San Diego, CA, USA) using the MiSeq v3 600-cycle reagent kit. Initial paired-end reads were quality trimmed using the Trimmomatic tool v0.33 $33^{34}$ and then, assembled by use of the de Bruijn graph-based de novo assembler SPAdes v3.14.0 $0^{35}$.

Map. Maps of the Czech Republic was created using the Leaflet package ${ }^{36}$ in R-studio ${ }^{37}$ from R-project ${ }^{38}$.

Long-read whole genome sequencing. Based on the results of short-read sequencing (see below), twenty-five KPC producers were selected to be sequenced using long-read sequencing technology, to help close the whole plasmid sequences. These isolates were selected as representatives of all different hospitals, bacterial species, STs, replicon profiles and KPC alleles.

Genomic DNA was extracted from the clinical isolates using NucleoSpin Microbial DNA kit (Macherey-Nagel, Germany). Whole genome sequencing (WGS) was performed on the Sequel I platform (Pacific biosciences, Menlo Park, CA, United States). Microbial multiplexing protocol was used for the library preparation according to the manufacturer instructions for Sheared DNA. DNA shearing was performed using the Megaruptor 2 (Diagenode, Liege, Belgium) using long hydropores producing $10 \mathrm{~kb}$ long inserts. No size selection was performed during the library preparation. The Microbial Assembly pipeline offered by the SMRT Link v9.0 software was used to perform the assembly and circularization with minimum seed coverage of 30X. Assembled sequences were annotated using the NCBI Prokaryotic Genome Annotation Pipeline (PGAP). 
Analysis ofWGS data. Antibiotic resistant genes, plasmid replicons and multilocus sequence types (MLST) were determined through uploading the assembled sequences to ResFinder 4.1 and CARD ${ }^{39}, 40$, PlasmidFinder ${ }^{41}$, and MLST $2.0^{42}$, respectively.

For sequence analysis, the BLAST algorithm (www.ncbi.nlm.nih.gov/BLAST), the ISFinder database (www-is. biotoul.fr/), and open reading frame (ORF) finder tool (www.bioinformatics.org/sms/) were utilized. Comparative genome alignment was done using Mauve v.2.3.1. (http://darlinglab.org/mauve/mauve.html) and BLAST Ring Image Generator (BRIG) ${ }^{43}$. Diagrams and gene organization were sketched using Easyfig v.2.2.2 ${ }^{44}$.

Transfer of $b l a_{\mathrm{KPC}}$-like genes. Conjugal transfer of $b l a_{\mathrm{KPC}}$-like genes from the clinical strains was carried out in mixed broth cultures ${ }^{45}$, using the rifampicin-resistant E. coli A15 laboratory strain as a recipient. Transconjugants were selected on MacConkey agar plates supplemented with rifampicin (150 mg/l) and ampicillin $(50 \mathrm{mg} / \mathrm{l})$. Transconjugants were confirmed to be KPC producers by PCR $^{11}$ and the MALDI-TOF MS meropenem hydrolysis assay ${ }^{30}$.

Phylogenetic analysis. Genetic diversity and phylogenetic relationship between the sequenced samples and global genomes were studied. All phylogenies were created using core genome, recombination and single nucleotide polymorphisms (SNPs) using parsnp v1.2, available in the harvest suite ${ }^{22}$ using a corresponding reference genome. SNPs identified in local collinear blocks were subsequently used for reconstructing an approximate maximum-likelihood tree using FastTree $2^{46}$ while including the general time reversible (GTR) model of nucleotide substitution. The Shimodaira-Hasegawa test implemented in FastTree2 was used to assess the support for significant clustering in the observed phylogeny. The interactive tree of life or iTOL (https://itol.embl. $\mathrm{de} /) .{ }^{47}$ was used for the graphic illustration of the trees along with relative annotations.

For the construction of the SNPs-based phylogenies, 582 Proteus mirabilis genomes were downloaded from NCBI assembly database including complete and draft genomes, using ASM6996v1 as reference. Similarly, 92 genomes for Morganella morganii (ASM1428397v1 as reference), 118 genomes for Citrobacter freundii (Cfr49,969 as reference), 126 genomes for Enterobacter hormaechei (Ecl-48,293 as reference) and 732 genomes for K. pneumoniae using Kpn-48,293 as reference.

Moreover, isolates from the study that clustered together forming a clade or/and subclade were investigated further. SNPs among the isolates within the clade/subclade were compared to a reference genome within the selected set using snippy v. 4.4 .3 (https://github.com/tseemann/snippy).

Nucleotide sequence accession numbers. The nucleotide sequence of the genomes and plasmids were deposited and available in GenBank under the BioProject number PRJNA700516; all accession numbers can be retrieved from Table S5.

Received: 10 May 2021; Accepted: 23 July 2021

Published online: 03 August 2021

\section{References}

1. Ambler, R. P. The structure of $\beta$-lactamases. Philos. Trans. R. Soc. Lond. B Biol. Sci. 289(1036), 321-331 (1980).

2. Queenan, A. \& Bush, K. Carbapenemases: The versatile $\beta$-lactamases. Clin. Microbiol. Rev. 20, 440-458 (2007).

3. Yigit, H. et al. Novel carbapenem-hydrolyzing $\beta$-lactamase, KPC-1, from a Carbapenem-resistant strain of Klebsiella pneumoniae. Antimicrob. Agents Chemother. 45, 1151-1161 (2001).

4. Chen, L. et al. Complete nucleotide sequences ofblaKPC-4- andblaKPC-5-Harboring IncN and IncX plasmids from Klebsiella pneumoniae strains isolated in New Jersey. Antimicrob. Agents Chemother. 57, 269-276 (2012).

5. Albiger, B., Glasner, C., Struelens, M., Grundmann, H. \& Monnet, D. Carbapenemase- producing Enterobacteriaceae in Europe: Assessment by national experts from 38 countries, May 2015. Eurosurveillance 20, 2 (2015).

6. Lee, C. et al. Global dissemination of carbapenemase-producing Klebsiella pneumoniae: Epidemiology, genetic context, treatment options, and detection methods. Front. Microbiol. 7, 2 (2016).

7. Grundmann, H. et al. Occurrence of carbapenemase-producing Klebsiella pneumoniae and Escherichia coli in the European survey of carbapenemase-producing Enterobacteriaceae (EuSCAPE): a prospective, multinational study. (2021).

8. Villegas, M. et al. First Identification of Pseudomonas aeruginosa isolates producing a KPC-type carbapenem-hydrolyzing $\beta$-lactamase. Antimicrob. Agents Chemother. 51, 1553-1555 (2007).

9. Cuzon, G. et al. Wide dissemination of Pseudomonas aeruginosa producing $\beta$-lactamase blaKPC-2gene in Colombia. Antimicrob. Agents Chemother. 55, 5350-5353 (2011).

10. Stoesser, N. et al. Genomic epidemiology of global Klebsiella pneumoniae carbapenemase (KPC)-producing Escherichia coli. Sci. Rep. 7, 2 (2017).

11. Naas, T. et al. Genetic structures at the origin of acquisition of the $\beta$-lactamase blaKPC gene. Antimicrob. Agents Chemother. 52, 1257-1263 (2008).

12. Bitar, I. et al. Interplay among IncA and blaKPC-carrying plasmids in Citrobacter freundii. Antimicrob.Agents Chemotherapy 63, 2 (2019).

13. Temkin, E., Adler, A., Lerner, A. \& Carmeli, Y. Carbapenem-resistant Enterobacteriaceae: Biology, epidemiology, and management. Ann. N. Y. Acad. Sci. 1323, 22-42 (2014).

14. Hrabák, J. et al. KPC-2-producing Klebsiella pneumoniae isolated from a Czech patient previously hospitalized in Greece and in vivo selection of colistin resistance. Folia Microbiol. 56, 361-365 (2011).

15. Hrabák, J. et al. Carbapenemase-producing Klebsiella pneumoniae in the Czech Republic in 2011. Eurosurveillance 18, 2 (2013).

16. Kukla, R. et al. Characterization of KPC-encoding plasmids from enterobacteriaceae isolated in a Czech hospital. Antimicrob. Agents Chemother. 62, 2 (2018).

17. Schweizer, C. et al. Plasmid-mediated transmission of KPC-2 carbapenemase in enterobacteriaceae in critically ill patients. Front. Microbiol. 10, 2 (2019). 
18. Majewski, P. et al. Emergence of a multidrug-resistant Citrobacter freundii ST8 harboring an unusual VIM-4 gene cassette in Poland. Int. J. Infect. Dis. 61, 70-73 (2017).

19. Hoffmann, H. \& Roggenkamp, A. Population genetics of the nomenspecies Enterobacter cloacae. Appl. Environ. Microbiol. 69, 5306-5318 (2003).

20. Papagiannitsis, C. et al. Characterization of KPC-encoding plasmids from two endemic settings, Greece and Italy. J. Antimicrob. Chemother. 71, 2824-2830 (2016).

21. Woodford, N., Turton, J. \& Livermore, D. Multiresistant Gram-negative bacteria: The role of high-risk clones in the dissemination of antibiotic resistance. FEMS Microbiol. Rev. 35, 736-755 (2011).

22. Treangen, T., Ondov, B., Koren, S. \& Phillippy, A. The Harvest suite for rapid core-genome alignment and visualization of thousands of intraspecific microbial genomes. Genome Biol. 15, 2 (2014).

23. GobeilleParé, S. et al. Arrival of the rare carbapenemase OXA-204 in Canada causing a multispecies outbreak over 3 years. J. Antimicrob. Chemotherapy 75, 2787-2796 (2020).

24. Hendrickx, A. et al. Plasmid diversity among genetically related Klebsiella pneumoniae blaKPC-2 and blaKPC-3 isolates collected in the Dutch national surveillance. Sci. Rep. 10, 2 (2020).

25. Leavitt, A., Chmelnitsky, I., Carmeli, Y. \& Navon-Venezia, S. Complete nucleotide sequence of KPC-3-encoding plasmid pKpQIL in the epidemic Klebsiella pneumoniae sequence type 258. Antimicrob. Agents Chemother. 54, 4493-4496 (2010).

26. Giakkoupi, P. et al. An update of the evolving epidemic of blaKPC-2-carrying Klebsiella pneumoniae in Greece (2009-10). J. Antimicrob. Chemother. 66, 1510-1513 (2011).

27. Studentova, V. et al. Complete nucleotide sequences of two NDM-1-encoding plasmids from the same sequence type 11 Klebsiella pneumoniae strain. Antimicrob. Agents Chemother. 59, 1325-1328 (2014).

28. Papagiannitsis, C., Miriagou, V., Giakkoupi, P., Tzouvelekis, L. \& Vatopoulos, A. Characterization of pKP1780, a novel IncR plasmid from the emerging Klebsiella pneumoniae ST147, encoding the VIM-1 metallo- $\beta$-lactamase. J. Antimicrob. Chemother. 68, 2259-2262 (2013).

29. Caltagirone, M. et al. Occurrence of extended spectrum $\beta$-lactamases, KPC-type, and MCR-1.2-producing enterobacteriaceae from wells, river water, and wastewater treatment plants in Oltrepò Pavese Area Northern Italy. Front. Microbiol. 8, 2 (2017).

30. Rotova, V., Papagiannitsis, C., Skalova, A., Chudejova, K. \& Hrabak, J. Comparison of imipenem and meropenem antibiotics for the MALDI-TOF MS detection of carbapenemase activity. J. Microbiol. Methods 137, 30-33 (2017).

31. Poirel, L., Héritier, C., Tolün, V. \& Nordmann, P. Emergence of oxacillinase-mediated resistance to imipenem in Klebsiella pneumoniae. Antimicrob. Agents Chemother. 48, 15-22 (2004).

32. Ellington, M., Kistler, J., Livermore, D. \& Woodford, N. Multiplex PCR for rapid detection of genes encoding acquired metallo-lactamases. J. Antimicrob. Chemother. 59, 321-322 (2006).

33. Yong, D. et al. Characterization of a new metallo- $\beta$-lactamase gene, blaNDM-1, and a novel erythromycin esterase gene carried on a unique genetic structure in Klebsiella pneumoniae sequence type 14 from India. Antimicrob. Agents Chemother. 53, 5046-5054 (2009).

34. Bolger, A., Lohse, M. \& Usadel, B. Trimmomatic: A flexible trimmer for Illumina sequence data. Bioinformatics 30, 2114-2120 (2014).

35. Bankevich, A. et al. SPAdes: A new genome assembly algorithm and its applications to single-cell sequencing. J. Comput. Biol. 19, 455-477 (2012).

36. Graul, Christian: leafletR: Interactive Web-Maps Based on the Leaflet JavaScript Library. R package version 0.4-0, (2016). at <http:// cran.r-project.org/package=leafletR>

37. RStudio Team RStudio: Integrated Development for R. RStudio, Inc., Boston, MA (2019) at <http://www.rstudio.com/>

38. R Core Team R: A language and environment for statistical computing. R Foundation for Statistical Computing, Vienna, Austria. (2020) at <https://www.R-project.org/>

39. Zankari, E. et al. Identification of acquired antimicrobial resistance genes. J. Antimicrob. Chemother. 67, 2640-2644 (2012).

40. Alcock, B. et al. CARD 2020: Antibiotic resistome surveillance with the comprehensive antibiotic resistance database. Nucleic Acids Res. https://doi.org/10.1093/nar/gkz935 (2019).

41. Carattoli, A. et al. In SilicoDetection and typing of plasmids using plasmidfinder and plasmid multilocus sequence typing. Antimicrob. Agents Chemother. 58, 3895-3903 (2014).

42. Larsen, M. et al. Multilocus sequence typing of total-genome-sequenced bacteria. J. Clin. Microbiol. 50, 1355-1361 (2012).

43. Alikhan, N., Petty, N., Ben Zakour, N. \& Beatson, S. BLAST Ring Image Generator (BRIG): Simple prokaryote genome comparisons. BMC Genom. 12, 2 (2011).

44. Sullivan, M. J., Petty, N. K. \& Beatson, S. A. Easyfig: A genome comparison visualizer. Bioinformatics 27(7), 1009-1010. https:// doi.org/10.1093/bioinformatics/btr039 (2011).

45. Vatopoulos, A., Philippon, A., Tzouvelekis, L., Komninou, Z. \& Legakis, N. Prevalence of a transferable SHV-5 type $\beta$-lactamase in clinical isolates of Klebsiella pneumoniae and Escherichia coli in Greece. J. Antimicrob. Chemother. 26, 635-648 (1990).

46. Price, M., Dehal, P. \& Arkin, A. FastTree 2-Approximately maximum-likelihood trees for large alignments. PLoS ONE 5, e9490 (2010).

47. Letunic, I. iTOL: Interactive Tree Of Life. Itol.embl.de (2021). at <https://itol.embl.de/>

\section{Acknowledgements}

We would like to thank the Czech participants of the "Working Group for Monitoring of Antibiotic Resistance" for providing the isolates. The participants information is detailed in Table S6 in supplementary materials.

\section{Author contributions}

C.C.P. and I.B. played an important role in interpreting the results and in writing the manuscript. K.C., V.J,. H.Z., V.S. and J.H. helped to acquire data. L.K., M.F., and I.B. carried out experimental work. I.B. and C.P. supervised the experiments and revised the final manuscript, which was approved by all authors.

\section{Funding}

The study was supported by research project grant NU20J-05-00033, provided by the Czech Health Research Council, by the Charles University Research Fund PROGRES (project number Q39), and by project CZ.02.1. 01/0.0/0.0/16_019/0000787 “Fighting Infectious Diseases," provided by the Ministry of Education Youth and Sports of the Czech Republic.

\section{Competing interests}

The authors declare no competing interests. 


\section{Additional information}

Supplementary Information The online version contains supplementary material available at https://doi.org/ 10.1038/s41598-021-95285-Z.

Correspondence and requests for materials should be addressed to C.C.P. or I.B.

Reprints and permissions information is available at www.nature.com/reprints.

Publisher's note Springer Nature remains neutral with regard to jurisdictional claims in published maps and institutional affiliations.

(c) (1) Open Access This article is licensed under a Creative Commons Attribution 4.0 International cc) License, which permits use, sharing, adaptation, distribution and reproduction in any medium or format, as long as you give appropriate credit to the original author(s) and the source, provide a link to the Creative Commons licence, and indicate if changes were made. The images or other third party material in this article are included in the article's Creative Commons licence, unless indicated otherwise in a credit line to the material. If material is not included in the article's Creative Commons licence and your intended use is not permitted by statutory regulation or exceeds the permitted use, you will need to obtain permission directly from the copyright holder. To view a copy of this licence, visit http://creativecommons.org/licenses/by/4.0/.

(C) The Author(s) 2021 